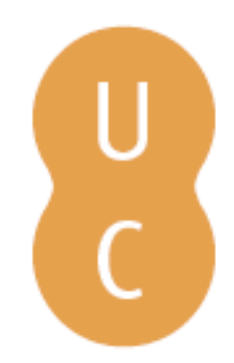

\title{
pommalina
}

\section{Selbst und Selbstbezug in der Kunst: zur Funktion des Ästhetischen in globalen Transferzusammenhängen}

\author{
Autor(es): $\quad$ Buchenhorst, Ralph \\ Publicado por: Imprensa da Universidade de Coimbra \\ URL \\ persistente: URI:http://hdl.handle.net/10316.2/31619 \\ DOI: $\quad$ DOI:http://dx.doi.org/10.14195/978-989-26-0205-9_4 \\ Accessed : $\quad$ 26-Apr-2023 14:02:41
}

A navegação consulta e descarregamento dos títulos inseridos nas Bibliotecas Digitais UC Digitalis, UC Pombalina e UC Impactum, pressupõem a aceitação plena e sem reservas dos Termos e Condições de Uso destas Bibliotecas Digitais, disponíveis em https://digitalis.uc.pt/pt-pt/termos.

Conforme exposto nos referidos Termos e Condições de Uso, o descarregamento de títulos de acesso restrito requer uma licença válida de autorização devendo o utilizador aceder ao(s) documento(s) a partir de um endereço de IP da instituição detentora da supramencionada licença.

Ao utilizador é apenas permitido o descarregamento para uso pessoal, pelo que o emprego do(s) título(s) descarregado(s) para outro fim, designadamente comercial, carece de autorização do respetivo autor ou editor da obra.

Na medida em que todas as obras da UC Digitalis se encontram protegidas pelo Código do Direito de Autor e Direitos Conexos e demais legislação aplicável, toda a cópia, parcial ou total, deste documento, nos casos em que é legalmente admitida, deverá conter ou fazer-se acompanhar por este aviso.

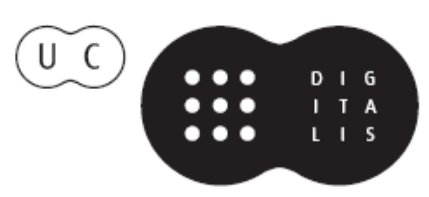


Edmundo Balsemão Pires

Burkhard Nonnenmacher

Stefan Büttner-von Stülpnagel

Editors

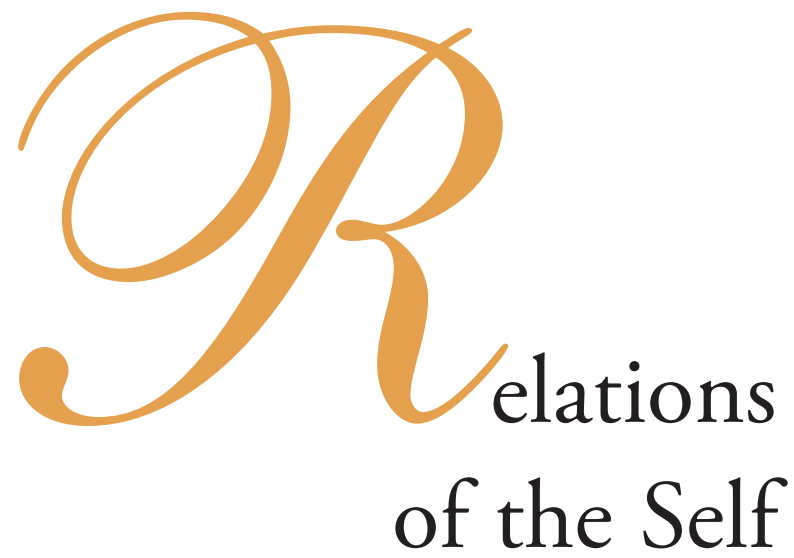




\section{Ralph Buchenhorst \\ Halle-Wittenberg}

\section{SELBST UND SELBSTBEZUG IN DER KUNST. ZUR FUNKTION DES ÄSTHETISCHEN IN GLOBALEN TRANSFERZUSAMMENHÄNGEN}

\section{Kontingenzen des Selbst ${ }^{1}$}

Wer über Selbstbezüglichkeit nachdenkt, der beschäftigt sich mit einem Begriff (und seinen Konsequenzen), der neben seiner Aktualität auch ein gewisses Querstehen zu gegenwärtigen gesellschaftlichen Prozessen mit sich bringt. Sicher ist das analytische Instrument der Selbstreferentialität für das Beschreiben von sozialen Entwicklungen in der Moderne unabdinglich, und die Vorstellung der Selbstbestimmung bleibt für das moderne Subjekt ein Grundpfeiler für sein Verständnis von Identität. Wir können nicht mehr, wie noch in der Vormoderne, uns und unsere sozialen Zusammenhänge so beschreiben, als würden die Vorbedingungen dieser Beschreibung aus einem Bereich ausserhalb des Gesellschaftlichen kommen, wie es in fundamental religösen Gemeinschaften der Fall war. Andererseits generieren wir Erkenntnis heute nicht mehr durch die selbstverständliche interne Evolution des je eigenen, hausgemachten (d.h. in europäischen Kategorien definierten) Wissens, sondern wir sind darauf angewiesen, externe Wissensbestände und Praktiken durch Adaptation, Transformation oder kritische (und dabei ex negativo Wissensteile integrierende) Ablehnung in die eigene Diskurskultur und Alltagspraxis einzuarbeiten. Dieses über Globalisierung hergestellte und kommunizierte Wissen greift nun seinerseits wieder in den Verlauf gesellschaftlicher Umbauprozesse ein. Das bringt in die gesellschaftlichen Selbstbeschreibungen eine wechselseitige Dynamik ein, die man vielleicht mit dem Konzept der Wanderung von epistemischen und normativen Ordnungselementen in neue Kontexte fassen könnte. Dieses Konzept muß sowohl den Einfluss sozialer Akteure als auch die Widerstände und Eigenlogiken der kreativen sozialen Praxis berücksichtigen, die mit der gesellschaftlichen Aufnahme wissenschaftlicher Deutungsvorgaben in Wechselwirkung stehen. ${ }^{2}$ Innerhalb dieses Konzepts

\footnotetext{
${ }^{1}$ Das im Folgende tragende Konzept der Translation verdankt sich den Diskussionen einer Forschergruppe der Martin-Luther-Universität Halle/Wittenberg, die Voraussetzungen sowie Folgen einer solchen konzeptionellen Vorentscheidung für die Beschreibung und Analyse von gesellschaftlichen Integrationsprozessen abzuwägen versucht. Insbesondere sei an dieser Stelle Richard Rottenburg und Matthias Kaufmann für ihre weitreichenden Kommentare und Anregungen gedankt.

${ }^{2}$ Vgl. Hans Joas, Die Kreativität des Handelns, Frankfurt/Main 1992
} 
macht die Vorstellung eines in traditionellen Kontexten aufgewachsenen und diese immer wieder reproduzierenden Selbst keinen Sinn mehr. Es wäre zu ersetzen durch die Vorstellung eines Selbst, das in permanentem Abgleich akzeptierter Werte und Orientierungen mit neuen kognitiven, ästhetischen und normativen Wissensangeboten sich zwar nicht als vollständig kontingent, aber doch als Resultat einer nicht im Ganzen kontrollierbaren Abgleichs von wechselnden Überzeugungskonstellationen begreift. Für dieses kontingente Selbst spielt die Einsicht, dass sein Bewußtsein das Resultat einer Mischung aus genetischer Präformierung und kulturspezifisch bedingter Erziehung ist, eine entscheidende Rolle. Die Lebensplanung des Einzelnen und sein Zugang zu kulturellem Kapital werden von traditionell strukturierten Kollektiven abgekoppelt und vollständig individualisiert. Die zweite Einsicht dieses Selbst besteht darin, dass das Oszillieren und Verschieben seiner Selbstbeschreibungen nicht stillgestellt werden kann, solange es sich der Kommunikation zwischen unterschiedlichen gesellschaftlichen Diskursen und individuellen Selbstreflexionen nicht völlig verschließt. Es wird permanent Zweifel an der Abgeschlossenheit dieser Beschreibungen hegen. ${ }^{3}$ Andererseits wird das Selbst innerhalb dieser Differenzerzeugung bestimmte Überzeugungen beibehalten wollen und sich von einer internen Logik der „Richtigkeit“ der Übersetzungs- und Integrationsprozesse leiten lassen. In der Hochphase der postmodernen Entwicklung hat der Architekturtheoretiker Charles Jencks den Pluralismus der Stile und Ausdrucksformen gegen einen Bedeutungsmonismus verteidigt: „No difference? - no richness, no meaning“. 4 Dem wäre heute hinzuzufügen, dass dieser Pluralismus solange eine bloße Juxtaposition bildet, wie die unterschiedlichen Ausdrucks- und Interpretationselemente nicht schlüssig (d.h anschlußfähig) übersetzt und lokalen Codes angepasst werden: No translation, no meaning.

Dies vorausgesetzt läßt sich folgerichtig behaupten, dass gegenwärtige soziale Wandlungen von heterogenen Formen der Wanderung und Verortung von Menschen, Artefakten und Modellen geprägt sind, die bereits durch ihre Quantität und die involvierten technischen Innovationen neuartige Herausforderungen darstellen ${ }^{5}$ und sich als Wanderung von Ideen zusammenfassen lassen, solange man davon ausgeht, dass dabei keine abstraktes metaphysisches Konstrukt unverändert und unveränderbar über Raum und Zeit transportiert wird. Die stattfindenden Übersetzungsprozesse setzen den notwendigen lokalen Anpassungen und Stabilisierungen sozialer Ordnungen neue Rahmenbedingungen, die zwar kontingenterweise zustande kommen, gleichwohl normative Funktionen übernehmen können. Denn die lokale Einbettung global zirkulierender Elemente durch Übersetzung führt zu strukturbildenden Wertekonstellationen und kognitiven Orientierungen und kann dadurch Innovationen auslösen, aber auch Widersprüche und Resistenzen ausprägen. Ursache dafür ist, dass lokale Wissensbestände, Praktiken und Repräsentationsformen immer häufiger und grundlegender in Widerspruch zu solchen Elementen normativer und epistemischer Ordnungen geraten, die für sich universale Geltung beanspruchen. Letztere legitimieren sich zumeist als Umsetzung

\footnotetext{
${ }^{3}$ Siehe dazu z.B. Richard Rorty, Kontingenz, Ironie und Solidarität, Frankfurt/Main 1992

${ }^{4}$ Charles Jencks, “Death for Rebirth”, in: A. C. Papadakis (Hrsg.), Post-Modernism on Trial, Architectural Design Profile No. 88, London 1990, S. 9

${ }^{5} \mathrm{Vgl}$. Anna Lowenhaupt Tsing, Friction: an ethnography of global connection, Princeton, N.J. 2005; Aihwa Ong und Stephen J. Collier (Hrsg.), Global assemblages: technology, politics, and ethics as anthropological problems, Malden, MA 2005
} 
weltkultureller Prinzipien und delegitimieren damit gleichzeitig partikulare nationale oder lokale Traditionen, ohne dass diese in der Alltagspraxis ganz ihre Wirksamkeit verlieren. Der Widerspruch führt dann oft zu dysfunktionalen hybriden Strukturen, die die Leistungsfähigkeit des Einzelnen sowie von Institutionen herabsetzen können, wie sich dies zum Beispiel bei der Umsetzung der Konsequenzen der PISA-Studie im Schulsystem und des Bologna-Prozesses an den deutschen Universitäten zeigt. ${ }^{6}$ Das Selbst muss sich in solchen Situationen entweder weitgehend von seinen traditionellen Bindungen an das gewachsene Kollektive lösen und unter Berufung auf die individuelle Autonomie globale Identifikationsmuster annehmen, oder aber in einer widerständigen Bewegung diese Muster als nicht authentisch ablehnen und seine eingelebten lokalen Praktiken bewußt stärken.

Will man diese Bewegungen beschreiben, dann muss man für die beobachteten Elemente von einer übersetzenden Wechselwirkung zwischen globalisierenden kulturellen und ökonomischen Netzwerken einerseits und gewachsenen historischen Kontexten andererseits ausgehen. Auf der Ebene des methodologischen Vorgehens, so behaupte ich, ist dabei das Konzept einer offenen Rationalität in Anschlag zu bringen, das von einem kontinuierlichen Austausch und Abgleich zwischen unterschiedlichen Rationalitätskonzeptionen ausgeht und von der permanenten Bereitschaft, vermeintlich Selbstverständliches in Frage zu stellen, wobei die Wissenschaftler, die dieses Rationalitätskonzept propagieren und zu fördern versuchen, seinen Anforderungen zu allererst selbst unterworfen sind. Offene Rationalität erfordert also ein hohes Maß an Selbstkritik. Gleichzeitig müssen die epistemischen Bedingungen für jenen Austausch und Abgleich vorgängig festgelegt werden, damit der Bezug zu einer inneren Logik des Argumentraums gegeben ist. Bei Kollisionen dieser Bedingungen im Rahmen der Austauschprozesse kann es jedoch dazu kommen, auch einzelne ihrer Elemente auszutauschen.

Zur Erläuterung dieses Ansatzes erwähne ich zwei Beispiele aus dem Bereich der Kunst, ein Bereich, der uns im Folgenden intensiver beschäftigen wird. Das erste Beispiel beschreibt die Integration der Form afrikanischer Masken in die europäische Kunstentwicklung. Als Picasso Anfang des 20. Jahrhunderts das Musée d'Ethnographie am Trocadéro in Paris besuchte und auf rituell verwendete Punu- und Fang-Masken aus Gabun stieß, führte das zu einer Umarbeitung seines bahnbrechenden Gemäldes Les Demoiselles d'Avignon (1907), das als Gründungswerk des Kubismus gilt. Das Translationsphänomen besteht darin, dass Picasso die ins ethnologische Museum überführten Masken, die damals in größerer Zahl durch Raub oder Kauf nach Europa gelangt waren, zur Lösung von ästhetischen Formproblemen verwandte. Picasso entwickelte diese Problemstellung aus dem von ihm radikalisierten Verständnis der Entwicklung der europäischen Formensprache, fand aber innerhalb ihrer keine adäquate Lösung dafür. Deshalb hatte für ihn die Konfrontation mit einer aussereuropäischen Formensprache eine revolutionäre Wirkung, die er im Werk selbst durch einen konkreten Formbruch ausdrückt. ${ }^{7}$ Allerdings setzte er das in und mit den Masken

\footnotetext{
${ }^{6}$ Vgl. zu dieser kritischen Einschätzung einer teils unreflektierten Überformung nationaler und lokaler Strukturen durch globale ökonomische und wissenschaftliche Eliten und deren Legitimationsmuster Richard Münch, Globale Eliten, lokale Autoritäten. Bildung und Wissenschaft unter dem Regime von PISA, McKinsey 6 Co., Frankfurt/Main 2009

${ }^{7}$ Dieser Bruch manifestiert sich im Vergleich der drei Figuren auf linken und der zwei auf der rechten Seite des Gemäldes. Die Gesichter der beiden Frauen rechts tragen Züge der erwähnten afrikanische Masken, sie scheinen einen direkten Bezug zur europäischen Formensprache zu verweigern. Die Gesichter der Frauen links lassen dagegen den Einfluss iberischer Formen erkennen, sie sind heller und weicher gestaltet.
} 
repräsentierte kulturelle Wissen nicht vollständig in seine eigene Arbeit um. Denn während die formalen Aspekte der Masken Eingang ins Bild fanden, blieben ihre rituelle Funktion und ihre semantische Komplexität im Kontext der Herkunftskulturen unberücksichtigt. Fremde Kulturelemente finden oft derart Eingang in die eigene, dass sie innerhalb der empfangenden Kultur selegiert, vereinfacht und auf aktuelle Problemlagen angewendet werden. Man wählt solche Elemente, die in eigene Legitimations- oder Ausdrucksmuster leicht integrierbar sind, wobei sich bei dieser Integration Sinnverschiebungen in der inneren Bedeutungsstruktur der Elemente ergeben. Afrikanische Masken z.B. lassen sich in ihrer Bedeutungsvielfalt oft gar nicht in Museen erkennen, weil ihnen der Kontext der spezifischen Bewegungsspiele, in die sie in den Ritualen faktisch eingebunden sind, fehlt. Ausserdem gibt es in bestimmten afrikanischen Sprachen kein Äquivalent für den abendländischen Begriff „Maske“. Angola-Masken in europäischen Museen sind oft nur auf das Kopfteil reduziert, das der europäische Besucher für die vollständige Camouflage hält, während ihren Produzenten und Anwendern selbst die gesamte Gesichts- und Körperbedeckung wiederum nur ein Aspekt dessen bedeutet, was besser nicht als Maske, sondern als Abbild eines Wesens aus der anderen Welt umschrieben werden könnte. ${ }^{8}$ Es findet also oft eine bloß partielle Rekontextualisierung eines fremden, sowohl ästhetisch als auch normativ lesbaren Strukturelements statt. Bewußte Akteure dieses Translationsprozesses wissen allerdings auch, dass der Erfolg oder die Wahrhaftigkeit dieser Rekontextualisierung sich nicht auf eine autochtone, ursprüngliche und nur durch diesen Ursprung autorisierte Bedeutung berufen kann. Sie halten auch diese Ursprungsbedeutung wiederum nur für einen aus einem Übersetzungsprozess heraus bestehenden und veränderbaren Sinnzusammenhang.

Als Indikator dafür, dass im Zuge einer Wanderung eine Translation stattgefunden hat, dient also nicht die möglichst getreue Wiedergabe eines Vorbildes, sondern vielmehr Belege dafür, dass zirkulierende Elemente in einem empirisch gegebenen Kontext (von Institutionen, Überzeugungsnetzwerken und technisch-materiellen Infrastrukturen) neue Verbindungen eingetreten sind, die wiederum bleibende Transformationen nach sich gezogen haben. Manche dieser so entstandenen Transformationen werden aus einigen oder den meisten Perspektiven eher wünschenswert, aus anderen hingegen eher problematisch erscheinen.

Das zweite Beispiel stammt aus dem Werk des argentinischen Erzählers und Lyrikers Jorge Luis Borges. Vielleicht gibt es keinen literarischen Text, der treffender den komplexen Umgang mit dem modernen Selbst beschreibt, als seine Kurzprosa mit dem Titel „Borges y yo“. Es handelt sich um eine Reflexion des Autors darüber, wer er selbst denn nun eigentlich sei, der öffentliche und sich über sein Schreiben definierende Schriftsteller Borges oder der Borges, der seinen privaten Alltag und seine persönlichen Vorlieben lebt. Das verwirrende Stück beginnt damit, dass der Autor des Textes, der private Borges, behauptet, es sei der andere, der Schriftsteller Borges, dem die Dinge eigentlich geschehen würden. Er selbst spaziere unbehelligt durch Buenos Aires, seine Architektur bewundernd, während er vom publizierenden Borges nur durch die Post oder ein biografisches Lexikon erfahre. Dann, gegen Ende des kurzen Textes, formuliert der Autor sein lakonisches Eingeständnis: Er werde sicherlich nicht verhindern können, dass irgendwann alle seine Erfahrungen an den

\footnotetext{
${ }^{8}$ Siehe dazu Kubik, G., „Afrikanische und europäische Transkulturation im Umfeld expressiver Kultur“, in: ders., Zum Verstehen Afrikanischer Musik. Aufsätze, Leipzig 1988, S. 322-336
} 
schreibenden Borges übergegangen sein werden. Aber er erkenne sich in dessen auf perverse Weise übertreibenden und fälschenden Büchern nicht mehr wieder als in vielen Büchern anderer Autoren. Die skurrile Prosa schließt mit dem irritierenden Satz: „Ich weiss nicht, wer von uns beiden diese Seite schreibt. ${ }^{\text {"9 }}$

Trotz der überraschenden Wendungen des Textes liegt es auf der Hand, welche Intentionen er verfolgt: Er vermittelt, dass man mit mehreren, nicht vollständig kompatiblen, gleichzeitig auch nicht eindeutig voneinander abgrenzbaren Identitäten leben kann. Er sagt auch, dass diese Identitäten von Kontingenzen durchzogen sind, die sie nicht zerstören, aber auch nicht vollständig kontrollierbar machen. Der in seinen Neigungen und Alltagsbedürfnissen lebende Borges geht in der Schriftstellerexistenz Borges scheinbar nicht auf, obwohl, wie am Ende des Textes angedeutet, die Tendenz zur Auflösung oder Abtretung sicherlich vorhanden ist. Vielmehr scheint der in seinen privaten sozialen Bezügen agierende Borges eine Facette der Existenz Borges auszumachen, die sein Schreiben nicht als ganze aufnehmen und repräsentieren kann. Borges problematisiert damit in diesem Text nicht nur, wie schon knapp zwanzig Jahre zuvor in seiner Erzählung „Pierre Menard, autor del Quijote ${ }^{\text {"10 }}$, den traditionellen Autor- und Textbegriff, und nimmt damit die poststrukturalistische Erzähltheorie vorweg. Er zeigt auch, dass Kunst eine Tätigkeit mit eigenen Gesetzen ist, die sich mit denen der Empirie nicht decken, obwohl die Empirie wiederum auf die Kunst angewiesen ist, damit sie in ihrer Fragilität sichtbar wird. Es handelt sich auch in diesem Fall um eine Art Übersetzung, die keine immer identischen Lebensentwürfe miteinander konfrontiert, sondern Teilbereiche des einen in den anderen versuchsweise integriert und dabei abwandeln muss.

Dieser Übersetzungsmechanismus stellt sich den Problemen der Kompatibilität einer Unzahl künstlicher Maschinensprachen und der Abgeschottetheit der Expertendiskurse untereinander als auch gegenüber der Alltagspraxis. Sie sind das Ergebnis des Ausdifferenzierungsprozesses von modernen Gesellschaften. Wir begegnen ihnen mit der wachsenden Produktion von Simultan- und Konsekutivübersetzungen. Analysen, die sich in unterschiedlichen und voneinander getrennten Diskursen auskennen, Übersetzungsprozesse transparent machen können und ausserdem die komplexe Beziehung dieser Fachsprachen mit dem Alltagsdiskurs nicht aus dem Blick verlieren, bilden ein Desiderat der Kultur- und Geisteswissenschaften. Sie bringen Prozesse in den Fokus, die Selbstreferentialität mit Angewiesenheit auf die Integration von fremden symbolischen und legitimierenden Elementen vermitteln. ${ }^{11}$ Eine Weise, diese Vermittlung zu thematisieren, ist die Frage, wie die autonome Kunstkommunikation sich auf Gesellschaft und auf die anderen ausdifferenzierten Diskurse bezieht. Damit bin ich bei der spezifischen Problematik angelangt, mit der sich die vorliegende Untersuchung im Folgenden beschäftigen will.

\footnotetext{
${ }^{9}$ Jorge Luis Borges, "Borges y yo", in: ders., Obras Completas, 12. Aufl. Buenos Aires 2002, Bd. II, S. 186, (übersettung von mir, R. B.)

${ }^{10}$ In: Borges, Obras Completas Bd. I, S. 444-450

${ }^{11}$ Als ein Beispiel der Übersetzung von verschiedenen Fachsprachen, hier der wechselseitigen Übertragung von naturwissenschaftlicher und ästhetischer Sprache: Michel Serres, Über Malerei. Vermeer - La Tour - Turner, Dresden/Basel 1995
} 


\section{Selbst- und Fremdbestimmung der Kunst}

Der beschriebene Gedanke Borges' ist, wir wissen das, der Ästhetik der Moderne nicht fremd. Die Kunsttheorie Adornos z.B. hat sich durchgängig mit dem Problem des Verhältnisses der Kunst zur sie umgebenden gesellschaftlichen Wirklichkeit auseinandergesetzt. Ihre analytische Struktur geht von einer zentralen Voraussetzung aus, die sie mit dem Begriff des Doppelcharakters der Kunst bezeichnet. Gemeint ist damit, dass die Kunstwerke paradoxerweise zugleich autonom und sozial determiniert sind - also zugleich selbst- und fremdbestimmt. Ästhetische Autonomie bei Adorno heißt Selbstseinkönnen des Werkes, Sichselbstbestimmenkönnen des Expressiven. Was im realen Leben bloße Selbsterhaltung ist, der Kampf des Subjekts mit den destruktiven Kräften von Natur und rigider Vergesellschaftung, wird in der Kunst zum Ideal des spielerischen Selbstseins der Werke. ${ }^{12}$ Denn das Subjekt genießt im Bereich der künstlerischen Formgebung Freiheiten gegenüber sich und den Dingen, die jenseits der Kunst dem Vorrang des Funktionalen, des Tauschwerts und der gesellschaftlich geforderten Konformität zum Opfer fallen würden. Diese Vorstellung prägt auch das Werk- und Materialverständnis Adornos. Selbstbestimmung bedeutet für ihn, dass die Werke sich ganz aus dem ihnen selbst innewohnenden ästhetischen Material auszubilden haben, dass sie sich ihre Stoffe und Techniken nicht von aussen vorgeben lassen dürfen. Durchbildung lautet dann der Begriff bei Adorno, der die Pflicht des Künstlers gegenüber dieser selbstbestimmten Formung des Materials bezeichnet. ${ }^{13}$

Andererseits ist dieses künsterische Ausagieren gesellschaftlich bedingt, also durch Fremdbezug definiert. Denn der Inhalt der Werke, auch der absichts- und inhaltslosesten, kommt nicht ohne das Moment eines Gemeinten, einer spezifischen Intention aus. Durch sie sind die Werke in gesellschaftliche Funktionszusammenhänge integrierbar, die den Werken selbst äußerlich bleiben. Die schlechten sind überdies dadurch korrumpiert, dass sie nicht jenem Ideal der immanenten Durchbildung des selbstgegebenen Materials folgen, sondern sich äußeren Einflüssen in naiver oder strategischer Weise öffnen. ${ }^{14}$ Die Instanz, die beide Momente, das des Autonomen und das des Fremdbestimmten, zur Reaktion bringt, ist das zugleich sich ästhetisch isolierende und sozial sich öffnende Subjekt. Man könnte auch sagen, dass dieses Subjekt zweigeteilt ist in ein ausserordentliches, kritisch reflexives Selbst und ein alltägliches, an Regeln der sozialen Ordnung gebundenes Selbst. ${ }^{15}$

Resultat dieser prekären, widersprüchlichen Konstellation des Subjekts bei Adorno ist die Einsicht in die Negativität der Kunst. Kunst bestimmt sich ausschließlich über das, was

${ }^{12}$ Theodor W. Adorno, Ästhetische Theorie, hrsgg. v. Gretel Adorno und Rolf Tiedemann, Frankfurt/Main 1973, S. 14

${ }^{13}$ Vgl. ebd., S. 313

${ }^{14}$ Das Veraltete dieses Materialbegriffs kritisiert Peter Bürger, Das Altern der Moderne. Schriften zur bildenden Kunst, Frankfurt/Main 2001, S. 10-30

${ }^{15}$ Kunstschaffen als ein Zusammenspiel dieser beiden Arten des Selbst zu konzipieren ist der Ansatz von Fred Orton, „Suspensa Vix Via Fit. Jasper Johns' Catenary: The Work of Art and the Art Object: The Everyday Self and the Extraordinary Self", in: J. Harris (Hrsg.), Value: Art: Politics. Criticism, Meaning and Interpretation after Postmodernism, Liverpool 2007, S. 275-300. Orton benutzt allerdings die Begriffe des alltäglichen und außerordentlichen Selbst eher im Sinne des frühen Heidegger 
sie nicht ist, und auf das ihr Äußerliche bezieht sie sich im Sinne der Zurückweisung und des Widerspruchs. ${ }^{16}$ Für Adorno heißt Negativität demnach nicht: Ende der Kunst, sondern: Selbstprovokation des Denkens. Unabgeschlossenheit ist dessen spezifisches Element. Adornos Kunstdenken geht immer wieder zwischen diesen Polen absoluter Selbstreferentialität und korrumpierender Fremdbestimmung hin und her, ohne sich auf einer der beiden Seiten oder bei einem Dritten zu beruhigen. Es lebt von dieser Bewegung. Mehr noch: Sie ist allererst das Existenzrecht dieses Denkens. Denn es ist gerade der philosophische Diskurs, der diesen Doppelcharakter der Kunst allererst erkennen kann. Die Kunst vollzieht ihn nur. Sie braucht Philosophie, um zu wissen, wie sie funktioniert. Man könnte von einer Fremdbeschreibung der Kunst durch die Philosophie sprechen. ${ }^{17}$

Ist aber zeitgenössische Kunst über dieses dialektische Element angemessen zu beschreiben? Ist sie konzeptualisierbar durch einen traditionellen, auf Kant und Hegel zurückgehenden Begriff des Selbst im Sinne der Bezugnahme auf Selbstbewußtsein und Reflexion ${ }^{18}$ Benötigt die Kunst überhaupt den philosophischen Diskurs, um ihres gesellschaftlichen Sinns habhaft zu werden? Oder ist sie auf eine Weise selbstreferentiell, dass ausschließlich in ihrem Innern definiert wird, was Kunst ist? Und, als weitere Frage: Benötigt die moderne Selbstauffassung die Idee einer freien Expressivität und einer unendlichen kritischen Reflexion, um Identität denken zu können? Ich werde im Folgenden versuchen, zwei Ansätze zu skizzieren, die sich als Antworten auf diese Fragen verstehen lassen.

\section{Zu den alternativen Quellen des Selbst}

Adorno hatte bereits in den 1920er Jahren von Walter Benjamin den Gedanken übernommen, dass naturhaftes Sein geschichtlich und zugleich geschichtliches Sein naturverhaftet ist. ${ }^{19}$ Dieser Gedanke wird im Hauptwerk der Frankfurter Schule, der Dialektik der Aufklärung von Horkheimer und Adorno, zur Aufforderung, der Natur im

${ }^{16}$ Zum Begriff der Negativität in der Ästhetik Adornos siehe Christoph Menke, Die Souveränität der Kunst, Frankfurt/Main 1991

${ }^{17} \mathrm{Zu}$ den Konsequenzen dieser Fremdbeschreibung siehe Gerhard Plumpe, Ästhetische Kommunikation der Moderne, 2 Bde., Opladen 1993

${ }^{18}$ Das Selbst ist ein Begriff des neuzeitlichen Denkens und seiner Subjektivierungstendenzen, hat also keine philosophische Vorgeschichte. In der Neuzeit wird im englischsprachigen Raum Descartes' Bestimmung des Geistes als res cogitans in das Konstrukt einer „conception of the self“ übersetzt. In der 1. Auflage der Kritik der reinen Vernunft charakterisiert Kant dann das reine, ursprüngliche, unwandelbare Bewußtsein, das als transzendentale Apperzeption (als spontane Vorstellung des „Ich denke“, die alle anderen Vorstellungen muß begleiten können) den Grund der Einheit des Bewußtseins bildet, als stehendes oder bleibendes Selbst im Flusse innerer Erscheinungen. Selbst ist hier also Selbstbewußtsein. Auch Hegel und Kierkegaard bestimmen Selbst noch durch die Bezugnahme auf das Bewußtsein und Reflexion. Erst Nietzsche bringt durch die Umwertung des Leibes ein neues Bestimmungselement des Selbst ins Spiel: „Werk- und Spielzeuge sind Sinn und Geist: hinter ihnen liegt noch das Selbst ... Es herrscht und ist auch des Ich's Beherrscher. Hinter deinen Gedanken und Gefühlen, mein Bruder, steht ein mächtiger Gebieter, ein unbekannter Weiser - der heisst Selbst. In deinem Leibe wohnt er, dein Leib ist er." (Friedrich Nietzsche, Also sprach Zarathustra, Kritische Studienausgabe, hrsgg. v. G. Colli/M. Montinari, Bd. 4, 2. Aufl. München und Berlin/N.Y. 1988, S. 39f.)

${ }^{19}$ Vgl. dazu Adornos Vortrag von 1932 „Die Idee der Naturgeschichte“, in: ders., Gesammelte Schriften Bd. 1, hrsgg. v. R. Tiedemann, Darmstadt 1997, S. 345-365 
Subjekt eingedenk zu bleiben. ${ }^{20}$ Die Autoren weisen darauf hin, dass das Subjekt nicht der autonomen Geschichte seines Selbstbewusstseins allein geschuldet ist, sondern wesentlich auch seiner Beziehung zur Natur, die es umgibt und die es in sich trägt. Daraus läßt sich ableiten, dass die Quellen des Selbst keinesfalls nur in der kognitiven und reflexiv-expressiven Entwicklung des Ichs liegen. Sie kommen auch aus der Natur, die das Subjekt qua Leib, also qua dem Einzelnen gegebenen und sinnlich wahrgenommenem Körper, ist. ${ }^{21}$ Dieser Körper manifestiert sich zum einen als kreative, das Bewußtsein überlistende somatische Instanz, etwa wenn durch Drogenexperimente das Bewußtsein als Kontrollinstanz geschwächt oder ausgeschaltet wird, um andere Wahrnehmungs- und Reaktionsweisen künstlerisch fruchtbar zu machen. Er wird aber eben auch als defizienter Modus des Daseins wahrgenommen, wenn er z.B. Schmerz verursacht oder grundnotwendige Funktionen nicht mehr ausüben kann. Seine Imperfektion macht dem Selbst dann spürbar, dass es einen Leib hat und mit dessen höchst unterschiedlichen Modi umgehen muss. ${ }^{22}$ Adorno hat versucht, dieses Gegenspiel von kreativer und defizienter Natürlichkeit des Selbst im Begriff der Idiosynkrasie zu fassen. Wir kennen solche Reaktionen. Scheinbar irrationale Überempfindlichkeiten gegenüber Gerüchen, Bewegungen, Zeichen und gesellschaftlichen Konventionen verweisen darauf, dass unser Körper sich gegen Fremdbestimmung wehrt. Dass dieses Sich-Zur-WehrSetzen nicht regressiv wird, also zu einem nostalgischen Sich-Zurück-Wünschen in unmittelbares Naturempfinden, verdankt das Subjekt für Adorno seiner Fähigkeit zu kritischer Selbstreflexion. ${ }^{23}$ Auch in dieser Auffassung zentriert sich also wieder jede Reaktion des Bewußtseins und des Körpers um die Idee der Autonomie, die das Subjekt verpflichtet, sich stets des fortgeschrittensten Stands der Produktivkräfte zu versichern und für seine Identitätsfindung gegenüber gesellschaftlicher Unterdrückung nutzbar zu machen.

Gegen diese Position ist von verschiedenen postmodernen Strömungen der Einwand geltend gemacht worden, sie bringe die Kunst unter den Zwang, permanent Neues schaffen, ihre eigene Geschichte also immer wieder entwerten zu müssen. Dass nur aus dieser Entwertung heraus Authentizität erstehen könne, bedeute eine Verarmung der ästhetischen Erfahrung. Man wies daraufhin, dass die Dichotomie von Gesellschaft und Individuum, von ökonomisch gesteuerten Machtinteressen und kulturellem Ich als Opfer dieser Macht, einer Ideologie geschuldet ist, die Gesellschaft nicht als komplexes Geflecht sich gegenseitig beeinflussender Entscheidungen versteht, sondern als strategisch agierendes Konstrukt ohne

${ }^{20}$ Max Horkheimer und Theodor W. Adorno, Dialektik der Aufklärung, Frankfurt/Main 1969

${ }^{21}$ So die These von Gernot Böhme, Ethik leiblicher Existenz, Frankfurt/Main 2008, S. $144 f f$.

${ }^{22}$ Peter Geimer hat in einem Artikel über den englischen Maler William Turner gezeigt, dass es neben den Würdigungen der ästhetischen Errungenschaften Turners auch Einschätzungen gab, die Turners späte Bilder als Resultate einer zunehmenden Linsentrübheit oder einer zerebralen Störung des Malers verstanden. Geimers Ausführungen machen klar, dass man die Grenze zwischen autonomer Ausübung kreativer geistiger Fähigkeiten und der Intervention des defizienten Körpers letztendlich nicht fixieren kann. (Peter Geimer, „Getrübte Blicke. William Turner in augenärztlicher Betrachtung", in: A. Mayer und A. Métraux (Hrsg.), Kunstmaschinen. Spielräume des Sehens zwischen Wissenschaft und Kunst, Frankfurt/Main 2005, S. 139-165)

${ }^{23}$ Vgl. Adorno, Ästhetische Theorie, S.69. Zum Verhältnis von Reflexion und Natur und zur Kritik des Begriffs einer naturbedingten Freiheit bei Adorno siehe auch Jürgen Habermas, „'Ich selber bin ja ein Stück Natur' Adorno über die Naturverflochtenheit der Vernunft", in: A. Honneth (Hrsg.), Dialektik der Freiheit. Frankfurter Adorno-Konferenz 2003, Frankfurt/Main 2005, S. 13-40 
Möglichkeit eines konkreten Horizontes von Verständigung. Aus dieser Kritik heraus entstand z.B. die Bewegung der Trans-Avantgarde, die dem künstlerischen Selbst eine Autorität zurückgewinnen wollte, die es ihm erlaubte, aus den Tiefen seiner eigenen Impulsivität und dem Formenreichtum der Kunstgeschichte wechselnde, moralisch nicht per se aufgeladene Bilder zu komponieren. ${ }^{24}$ Diese Wendung gab allerdings auch den (indirekten) moralischen Anspruch der modernen Kunst, gesellschaftliche Verantwortung bei der Formgebung immer mitzuberücksichtigen, preis und zog sich auf die unhintergehbaren inneren Antriebe des Selbst als letztgültige Instanz der Kunstproduktion zurück.

Ich möchte nun zeigen, wie sich der rigide Subjektbegriff Adornos auflösen läßt, ohne den moralischen Appell des sozial verankerten Selbst völlig aufgeben zu müssen. Gernot Böhme, Technikphilosoph und Verfechter einer ökologischen Naturästhetik, wendet sich gegen das veraltete Ideal eines autonomen Subjekts bei Adorno und bringt stattdessen einen Begriff des Selbst ins Spiel, in dem Verletzlichkeit, physische Unvollkommenheit und Angewiesensein auf seine eigene Kreatürlichkeit entscheidende Momente darstellen. In dieser Verletzlichkeit wird das Selbst zu einer Instanz, die seine eigene, unvollkommene Natürlichkeit akzeptieren und in seinen Lebensentwurf integrieren muss. Es wird zu einem Subjekt, so Böhme, das „nicht Herr im eigenen Hause ist“. ${ }^{25}$ Dass das Subjekt ohne seine Naturgebundenheit nicht gedacht werden kann, bedeutet also nicht, dass diese Gebundenheit permanent durch Selbstreflexion und Anerkennung kritisch zu überwinden ist. Es bedeutet vielmehr, dass Erleiden konstitutiv zum Selbst dazugehört. Böhme ersetzt so die Vorstellung des autonomen Subjekts durch die des souveränen Menschen. Dieser nimmt sein Selbst nicht nur als reflektiertes Handeln, sondern auch als bewußtes Erleiden wahr, als das Erleiden der Unvollständigkeit, des Nicht-sich-selbst-Genügen des als Selbstbewußtsein gedachten Subjekts.

Die Konsequenzen, die er aus dieser Umorientierung bezüglich der Quellen des Selbst zieht, sind vornehmlich ethischer Natur. ${ }^{26}$ Böhme schreibt schon dem menschlichen Leib Würde zu, nicht erst dem Menschen als gesellschaftlichem oder von Gott gemachtem und gesegnetem Wesen. Menschenwürde werde bereits verletzt, wenn basic needs wie Grundernährung, Obdach oder Geschlechtsverkehr nicht befriedigt werden können. ${ }^{27}$ Seine ethischen Folgerungen möchte ich aber auch für unsere ästhetischen Überlegungen fruchtbar machen. Mir geht es also nicht so sehr um die normativen Konsequenzen jenes Erleidens für den bewußt wahrgenommenen Körper und den gesellschaftlichen Umgang mit ihm, sondern um die Konsequenzen des Nicht-sich-selbst-Genügens der Kunstproduktion im autonom gedachten Subjekt. Die Böhmsche Idee des Ungenügens inspiriert, über dasjenige des Werks und der Künstlerfigur nachzudenken. Denn zeitgenössische Kunstreflexionen gehen, so scheint mir, nicht mehr von dem Ideal einer autonomen Kunst im Sinne einer fortgeschrittenen Selbstbestimmung des einzelnen, aus befreiter Expression gemachten Künstlersubjekts aus.

${ }^{24}$ Vgl. z.B. Achille Bonito Oliva, „Die italienische Trans-Avantgarde“, in ders., Im Labyrinth der Kunst, Berlin 1982, S. 54-100

${ }^{25}$ Vgl. Böhme, Ethik, S. 149

${ }^{26}$ Es war vor ihm bereits Freud, der darauf hinwies, dass unser Moralgefühl nicht das Ergebnis einer vorempirischen, absoluten moralischen Instanz, sondern dass es genauso idiosynkratisch ist wie die formalkreativen Reaktionen des ästhetischen Subjekts

${ }^{27}$ Vgl. ebd., S. $18 \mathrm{ff}$. 
Sicher ist Kunst autonom in dem Sinne, dass es der ästhetische Diskurs allein ist, der bestimmt, was als Kunst gilt und was nicht. Und wenn die Kunst als esoterischer Sonderdiskurs infragegestellt oder ihr Ende propagiert wird, so sind das Tendenzen, die im Inneren dieses Diskurses selbst erzeugt, diskutiert und dann ironisch verfeinert werden. ${ }^{28}$ Aber diese Autonomie hat ihren Sitz nicht allein im Objekt oder im autorisierenden Künstler. Sie entfaltet sich außerdem in der Relation zwischen diesen und den Betrachtern. ${ }^{29}$ Das bedeutet, dass Bedeutung nicht im Werk entsteht, und Expressivität nicht allein im Agieren und Produzieren des Künstlers, sondern dass Beides erst im Zwischenraum zwischen Werk, Ausstellungsort und Betrachtern (im Plural!) sich bildet und immer wieder neu erzeugt werden muß. Die Selbstbeziehung der Kunst manifestiert sich hier also im Sinne eines Abgleichs verschiedener symbolischer Ordnungsprinzipien und Verstehensmuster, der erst in der performativen Situation der ästhetischen Erfahrung im kollektiven Raum der Begegnung von Produzenten, Institutionen, Interpreten und historischen Konstellationen zustande kommt. Die Unabschließbarkeit dieses Prozesses ist nun durch das Oszilieren zwischen bloßem Ding und reinem Zeichen nicht zureichend bezeichnet. Sie ist jenem eingangs geschilderten Prozess der Übertragung geschuldet. Nur indem jener Abgleich in Räumen stattfindet, die eine permanente Migrations- und Übertragungsleistung von symbolischen, expressiven und normativen Elementen ermöglichen, macht den Bedeutungsfindungsprozess zugleich unabschließbar und nachvollziehbar. Kunstproduktion und -interpretation ist die Konstituierung von ästhetischem Sinn aus den Vermittlungsleistungen, die offene Diskurse heute in bedeutungsrelativen Umwelten permanent erzeugen. Unhintergehbare lokale Bedingungen und global geltende kommunikative Muster gestalten sie in gleicher Weise mit. Sie entfalten nicht mehr einen Begriff des Menschen im Sinne eines homo creator mit dem Ziel einer Selbstüberwindung oder dem Ziel der Umsetzung der verfügbaren ästhetischen Produktivkräfte im Werk. Vielmehr werden Zeitlichkeit, Performativität, die Einbeziehung der Körperlichkeit des expressiven Machens und die dezentrierende Perspektive der historisch, regional und normativ bedingten und damit eingeschränkten Sinnfindung ins Zentrum der Überlegungen gestellt.

Dieses ästhetische Konzept ermöglicht auch eine intersubjektive und interkulturelle Umdeutung des Idiosynkratischen als einer Quelle des Selbst. Es wäre so nicht mehr an die konfliktive Beziehung des Einzelnen zur Natur und zum Mimetischen gebunden, sondern an die diskursiven und intuitiven Schwierigkeiten einer Kultur oder einer gesellschaftlich ausgeprägten Ausdrucksleistung, symbolische und normative Ordnungselemente anderer Kulturen oder Diskurse in die eigenen Ausdrucks- oder Erklärungsmuster zu integrieren oder sich von ihnen zu distanzieren. Das Allgemeine der Kunst wäre dann nicht durch die Idiosynkrasie des Einzelnen hindurch zu entwickeln, sondern in jenem permanenten Abgleich zwischen geformtem Ding, seinem ihn umgebenen Raum, und einem prekären Geflecht zugänglicher und verstehbarer Interpretationsprinzipien. Die Selbstbeziehung des

\footnotetext{
${ }^{28}$ Siehe zu diesen Tendenzen z.B. Arthur C. Danto, After the End of Art. Contemporary Art and the Pale of History, Princeton 1997; Eva Geulen, Das Ende der Kunst. Lesarten eines Gerüchts, Frankfurt/Main 2002; Hans Belting, Das Ende der Kunstgeschichte. Eine Revision nach zehn Jahren, 2. Aufl. München 2002

${ }^{29}$ Vgl. dazu „Zur Aktualität ästhetischer Autonomie. Juliane Rebentisch im Gespräch“, in: Inaesthetik. Theses on Contemporary Art, Nr. 0, Juni 2008, hrsgg. v. T. Huber und M. Steinweg, S. 103-118
} 
Subjekts, die nur durch die Identität des sich selbst erfahrenen und expressiven KünstlerIchs gedacht werden kann, wird durch eine Beziehung ersetzt, die die Selbsterzeugung der Kunst durch die permanente Reinterpretation von expressiven Räumen denkt, und diese Reinterpretation wird notwendigerweise gespeist durch eine Reorganisation von symbolischen und epistemischen Ordnungsmustern, die sich der interkulturellen Kommunikation in globalen Bezugsräumen stellt.

In einer Ereignisästhetik z.B. tritt das Handeln und Entscheiden des Subjekts, seine Intentionalität, in den Hintergrund. Aspekte der Responsivität, der Unverfügbarkeit und des Erleidens werden grundlegend. ${ }^{30}$ Wenn man das Kunstsystem als selbstreferentielles versteht, also als eine Produktion und Rezeption von Expressionen, die selbst bestimmen, was Kunst ist, dann werden Elemente, die sich weder als fortschrittlich noch als durchgebildet verstehen, trotzdem noch als Kunst wahrgenommen und akzeptiert. In der Musik sind das Weghören oder ein defizientes Hören, niedrige und populäre Kompositionstechniken wie die plunderphonics heute approbierte ästhetische Elemente. ${ }^{31}$ Ich nenne zwei weitere Beispiele aus der zeitgenössischen Kunstproduktion und eines aus der Aufführungspraxis mittelalterlicher Musik.

\section{Performative Öffnung der Kunst}

Der Künstler Jochen Gerz hat in einer Aktion, der Bremer Befragung: Sine somno nibil (1990-1995), statt eines fertigen und vom Künstler per Signatur autorisierten Werks eine interaktives Entscheidungsspiel initiiert. Gerz führte zusammen mit Bremer Kunststudenten eine Umfrage durch, bei der 50.000 Bürgern der Stadt folgende Fragen gestellt wurden:

- Zu welchem Thema sollte das Bremer Kunstwerk Stellung nehmen?

- Glauben Sie, dass es leicht ist, Ihren Vorschlag mit den Mitteln der Kunst zu verwirklichen?

- Kann die Kunst Probleme lösen? Und welche Probleme sollte sie Ihrer Meinung nach lösen können?

- Möchten Sie an dem Bremer Projekt mitarbeiten? ${ }^{32}$

Die Teilnahmewilligen wurden dann zu sechs öffentlichen Seminaren eingeladen, die mit der Erklärung endeten, dass öffentliche Kunst keines materiellen Objektes bedarf. Der Betrachter soll nicht Kunst im Werk eines einzelnen Autors ablesen, sondern als Partizipierender das Werk selbst mitschaffen. Seine Erwartungshaltung, die mit einem fertigen Kunstprodukt rechnet, dem man dann nachträglich mit Kritiken oder distanziertem Misstrauen begegnen kann, wird enttäuscht. Damit wird er in der Frage, was Kunst sei und welche Kunst er wolle, immer auf sich selbst zurückgeführt. Diese Konstellation, für die Gerz den von Joseph Beuys in

${ }^{30}$ Siehe als Beispiele: Dieter Mersch, Ereignis und Aura. Untersuchungen zu einer Ästhetik des Performativen, Frankfurt/Main 2002; Eva Fischer-Lichte, Ästhetik des Performativen, Frankfurt/Main 2004

${ }^{31}$ Vgl. Tobias Plebuch, „Musikhören nach Adorno. Ein Genesungsbericht“, in: Merkur Heft 8, 56. Jg., August 2002, S. $675-687$

${ }^{32}$ Vgl. Jochen Gerz, Die Bremer Befragung: Sine somno nihil, Ostfildern 1995 
die zeitgenössische Kunst eingeführten Begriff der Sozialen Plastik wählt ${ }^{33}$, ist mit den theoretischen Mitteln der ästhetischen Hochmoderne, wie sie Adorno entwickelt und benutzt, nicht angemessen zu erklären. In ihr ist der Publikumsbegriff radikal verändert. Er repräsentiert nicht mehr Gesellschaft, und der Künstler repräsentiert nicht mehr die Autorität, die das Werk erst zur Kunst macht. Weil der einzelne Beobachter selbst auf die ästhetisch definierte Oberfläche einwirkt, erhalten die beiden grundlegenden Relationen der Kunst einen offenen Charakter: diejenige zwischen ästhetisch aufbereitetem Material und Beobachter und diejenige zwischen Geschichte und manifester Spur. ${ }^{34}$ Auf diese Weise kann Kunst offene Formen konzipieren, die ihren eigenen Begriff transformieren. Ihre Frage nach der Gegenwart von Geschichte zusammen mit dem bewussten Einbeziehen von Ko-Autoren eröffnet ihr diskursive Möglichkeiten jenseits des autorisierten Meisterwerks oder des bloßen Entertainments. ${ }^{35}$

Diese Entwicklungen haben weitere Konsequenzen für die Beziehung zwischen Autor und öffentlichem Raum. Die Konstitution eines kollektivnachvollziehbaren sach - und Ausdruckgehalts im Werk erfordert nicht nur die permanente Kommunikation zwischen Künstler und (noch als Einzelnem gedachten) Betrachter, die ins Werk selbst eingehen muss, sondern automatisch damit auch die Auseinandersetzung mit der sich ändernden Stellung von Autor und Werk im (geopolitisch gedachten) öffentlichen Raum. Sollen sich jene Gehalte im Werk kollektiv konstituieren, müssen sie dort nicht nur derart manifest werden, dass die Spuren jener Auseinandersetzung ins Werk einfließen, sondern der Autor selbst muss sich im öffentlichen Raum so situieren, dass er seine Identität nicht mehr ausschließlich über einen inneren Monolog definiert, sondern in der konkreten Auseinandersetzung mit einer sich globalisierenden Öffentlichkeit. Die Identität des Autors ist somit nicht mehr herstellbar über die Frage nach Intuition, technischer Fertigkeit und individuellem Innovationspotential, sondern konstituiert sich im sozialen Zusammenspiel der zufällig oder intendiert, konkret oder potentiell Teilnehmenden. Nur diese Öffnung ermöglicht es einerseits dem Betrachter, seine bloß reaktive Funktion aufzugeben und eine aktive Position in der Konstruktion des Werkes zu gewinnen, und andererseits dem Künstler, sich aus seinem Rückzug auf sich selbst als der Autorität, deren Vorstellung von Pluralität und Kommunikation alleine gelten soll, zu befreien. Verfolgt er diesen Weg nicht, besteht die Gefahr, in ein Verhalten zu verfallen, mit dem wir „nur noch auf Sicherheit aus sind, Ordnung, Struktur, bis wir jede Reziprozität fürchten “36.

${ }^{33}$ Man macht eben keine Skulptur oder Plastik für die Gesellschaft, sondern diese selbst ist eine Plastik: „Wir sind das Defizit, nicht die Kunst. Wir müssen sehen, ob wir nicht mehr sein können.“ (Vgl. Jochen Gerz, „Soziale Plastik heute“, in: J. Jäger und P.-K. Schuster (Hrsg.), Das Ende des XX. Jahrhunderts. Standpunkte zur Kunst in Deutschland, Köln 2000, S. 107-119, Zitat S. 118)

${ }^{34}$ Im Sinne der Suggestionen Gerzens: „Die Zeit schließt wieder zu den Objekten auf.“, „Der Autor wird im Kontext gesehen.“, „Das Werk muß modifizierbar werden im Lesen.“ (Jochen Gerz, „For the second time - a call for modernity“, in: H. Klotz (Hrsg.), Die Zweite Moderne. Eine Diagnose der Kunst der Gegenwart, München 1996, S. 90-100)

${ }^{35}$ Der Rückzug der Kunst auf letztere Funktion wird von vielen Theoretikern ausgerufen und bildet seit über drei Jahrzehnten eine Front, die das Ende der Moderne in ihrer radikalen Form ausruft, vgl. z.B. Donald Kuspit, The end of art, Cambrigde 2004, S. 14, S. 175ff.; Hans Peter Schwerfel, „Ground Zero und Stunde Null“, in: ders. (Hrsg.), Kunst nach Ground Zero, Köln 2002, S. 9-13

${ }^{36}$ Gerz, For the second time - a call for modernity, S. 97 
Als weiteres Beispiel möchte ich das Helicopter String Quartet von Karl-Heinz Stockhausen (1992/93) anführen. Es steht für die Einbeziehung der Körpernatur als Quelle des ästhetischen Selbst. Stockhausen komponierte das Streichquartett in ein Ensemble von vier fliegenden Hubschraubern hinein, in die sich die vier Ausführenden begeben, wobei sie über Mikrofone und Kopfhörer miteinander kommunizieren können. Musik und Bild erreichen das Publikum am Boden über Videokameras und installierte Bildschirme. Das Werk repräsentiert zum einen den Traum, den menschlichen Körper nicht nur klang-, sondern auch flugfähig zu machen. Die spezifischen Modalitäten, Wünsche und Defizienzen des Körpers fließen ein in diese Kunst, die den akustisch optimierten Konzertsaal verlässt und sich mit kunstfremder Technik und der Beziehung des menschlichen Körpers zu ihr auseinandersetzen muss. Sie konfrontiert damit zum anderen den hermetisch abgeriegelten klassischen Klangraum, in dem das Publikum die Rolle des schweigenden, disziplinierten Rezipienten übernommen hat, mit dem Raum und der Funktion eines geräuschvollen, urbanen oder militärischen Transportmittels. Die Bedeutung jenes abgedunkelten, Ehrfurcht erregenden Theater- und Konzertraums in der bürgerlichen Gesellschaft des 19. und beginnenden 20. Jahrhunderts bestand vor allem darin, die Selbstinszenierung des vortragenden Künstlers zu ermöglichen, also die vollständige Konzentration und Isolation des unbedingten Ausdruckswillens. Das Publikum hatte dabei nur die Funktion der schweigsamen, disziplinierten Aufnahme des Dargebotenen. ${ }^{37}$ Diese Hermetisierung und Heroisierung des Künstlers besteht in den großen Aufführungssälen weltweit fort und ist ein Grund dafür, im Komponisten und aufführenden Interpreten weiterhin nur durch sich selbst autorisierte Schöpfer zu sehen. Der sogenannte „performative turn“ in den Kulturwissenschaften ${ }^{38}$ betont zwar eine seit Beginn des 20. Jahrhunderts zunehmende Theatralisierung des öffentlichen Raums und in der postindustriellen Gesellschaft ein neues Bewußtsein für das Rituelle und Interaktive bei Großveranstaltungen ${ }^{39}$, jedoch scheint dies mehr ein theoretisches als ein verbreitetes aufführungspraktisches Phänomen zu sein.

Das Helicopter String Quartet bestätigt zwar die Position der einzelnen, sich selbst legitimierenden Komponistenfigur, setzt aber für die Aufführungspraxis auf sehr anschauliche und konkrete Weise das Übertragungsprinzip in Szene: Die musikalische Umsetzung kann nur durch technische Transmitter hörbar gemacht werden, die komponierte Musik steht in einem direkten Vermittlungszusammenhang mit der durch die Rotorblätter und die Motoren erzeugten Geräuschkulisse, und schließlich findet eine Übersetzung eines klassischen europäischen Klangkörpers und Genre (das Streichquartett) des 18. Jahrhunderts in einen Klang- und Bewegungsraum statt, der unmittelbar dem 20. Jahrhundert angehört. Nicht unerheblich für das kritische Potential dieser Komposition ist auch, dass die ausführenden

\footnotetext{
${ }^{37}$ Vgl. zu dieser Entwicklung Richard Sennett, Verfall und Ende des öffentlichen Lebens. Die Tyrannei der Intimität, Frankfurt/Main 1986, S. $264 \mathrm{ff}$.

38 Als Überblick dazu siehe Doris Bachmann-Medick, Cultural Turns. Neuorientierungen in den Kulturwissenschaften, Reinbek bei Hamburg 2006, S. 104-143

${ }^{39}$ Vgl. Eva Fischer-Lichte, „Theater als Modell für eine performative Kultur. Zum performative turn in der europäischen Kultur des 20. Jahrhunderts“ (Vortrag am 28.01.2000), Saarbrücken: Universitätsreden der Saarländer Universität 46
} 
Musiker bei der Interpretation des Werkes in sehr konkreter Weise auf die Flugkünste der steuernden Hubschrauberpiloten angewiesen sind.

Freilich sollte auch darauf hingewiesen werden, dass Übersetzungsprozesse scheitern können oder den Geschmack des Mißtrauens oder der Unzufriedenheit hinterlassen. Meistens liegt diese Einschätzung dann vor, wenn eine symbolische Ordnung, in die übersetzt werden soll, so grundlegend verschieden strukturiert ist, dass man zu viele Einbußen für die Komplexität des Sinnzusammenhangs im Übertragungsvorgang hinnehmen muß. Einen Fall für dieses Problem bieten die Fragen einer heutigen Aufführungspraxis für mehrstimmige Musik des 11. und 12. Jahrhunderts. Es existieren z. B. überlieferte Notationen für polyphone Gesänge aus dem Umkreis der Abtei St. Martial-de-Limoges. Diese Musik gilt als frühes Beispiel mehrstimmig komponierter Musik in Westeuropa. Für sie gibt es keine eindeutig definierten Regeln zur Aufführung, unter anderem weil die handschriftlichen Quellen des St. Martial-de-Limoges Repertoires nur schwer zu entziffern sind und viele der Zeichen noch nicht eindeutig bestimmt werden konnten. Es gibt nun Auffassungen, die den heutigen Sinn dieser Musik sich eher in einer performativen Herangehensweise entfalten sehen, d. h., sie von der historischen Notationsvorlage durch die kommunikative Übepraxis direkt umzusetzen, ohne den Umweg einer Übertragung in unser approbiertes modernes Notationssystem zu nehmen. Grund dafür ist, dass die mittelalterlichen Neumen (also das graphische System der Notation der melodischen Gestalt mittelalterlicher Gesänge) die konkrete Musik anders in ein symbolisches System umsetzten. In diesen Aufzeichnungen findet man unzählige Symbole, die Anhalten, langsames oder beschleunigtes Durchgehen, Tremolieren, Vibrieren, Bebungen der Stimme, Verschleifungen von Tönen und anderes anzeigen. Daraus läßt sich schließen, wie reich ein Vortrag gestaltet wurde, aber auch, wieviel wohl nicht notiert wurde, obwohl es zum Usus der Interpretation gehörte.

All dies müßte man in moderne Notation übersetzten, indem man neue Zeichen dafür erfindet. Doch der Gewinn einer solchen Übertragung erscheint fraglich. Denn man kann sich durchaus ausschließlich auf die originale Notation verlassen, in der eine Vielzahl wichtiger musikalischer Parameter bestens aufgezeichnet ist. Eine Übertragung ins heutige System kann zwar einerseits die Umsetzung für solche Musiker erleichtern, die in mittelalterlicher Musikpraxis nicht sehr geübt sind, gaukelt aber damit eben eine falsche Vertrautheit mit dem musikalischen Material vor. Zudem suggeriert die Umschreibung in moderne Notation auch eine gesicherte, unabhängige Speicherung der musikalischen Substanz, während eine rudimentäre historische, nur andeutende Notation den Hinweis auf die Wichtigkeit der Memorierleistung in der Musikpraxis des Mittelalters erhält. Diese Verweigerung der Übersetzung relativiert unsere heutige Schriftfixiertheit. Die Ambivalenz von Schrift wird deutlich, da die spätere, komplexere Notationen gegenüber früheren durchaus auch einen Verlust bedeuten kann. ${ }^{40}$

Diese Bedingungen können den Einzelnen also am Ende dazu veranlassen, die mittelalterliche Notation auf andere Weise zu übersetzen als durch eine Translation in das moderne Notationssystem. Wahrscheinlich hilft dabei nicht nur eine profunde Kenntnis der historischen Entwicklung der abendländischen Musik und Musiknotation vom Mittelalter

\footnotetext{
${ }^{40}$ Vgl. Ellen Hünigen, Noster Cetus Psallat Letus. Aquitanische Polyphonie des 12. Jahrhunderts, unveröffentlichtes Manuskript 2008.
} 
bis in die Gegenwart, sondern auch die Kenntnis von nicht schriftfixierten Kompositionsund Aufführungspraktiken außereuropäischer Kulturen. Auch hier gilt also am Ende wieder, dass die Interpretation eines symbolischen Systems ohne Übersetzungsleistungen nicht auskommt, da diese Kenntnisse erst in den aktuellen Stand der Aufführungspraxis integriert werden müssen. Für das Selbst hat die eben beschriebene Konstellation die Konsequenz, dass es sich nicht auf ein autorisiertes, alle musikalischen Parameter genau bestimmendes Schriftsystem beziehen kann, sondern in der ad hoc sich vollziehenden Interpretation und Umsetzung eine nicht umgehbare Unbestimmtheit akzeptieren muss. Das Selbst kann sich also nicht dem Bedeutungskörper der Notation rückhaltlos überlassen, sondern muß in einer prozesshaften, die Dimension der kontextabhängigen Selbstgestaltung betonenden Leistung eine bewußt ungesicherte ad-hoc-Setzung vornehmen. Man darf also auch hier von einer performativen Öffnung des ästhetischen Prozesses sprechen. Denn jene Leistung ist nicht bestimmt durch die Verhaltensmuster der Authentizität und historische Treue, sondern durch das der Resonanz, d.h. durch das Ereignis des Antwortens auf eine unvollständige Schriftanweisung und auf das intuitiv bewertete Klangresultat des Kollektivs. ${ }^{41}$

\section{Selbstreferentialität des Kunstsystems}

Kommen wir nun zu dem zweiten Ansatz, den ich Ihnen versprochen habe. Er soll die Frage beantworten, ob Kunst nur von einem Anderen her, dem dialektischen Denken, zu verstehen ist, oder ob ihre Selbstreferentialität derart ausgeprägt ist, dass nur von und in ihr selbst deutlich wird, was Kunst ist. Das dialektische Denken hat ja eine ethische Implikation, die es selbst nicht rechtfertigen kann. Es bestimmt ernsthafte, avancierte, funktionslose Kunst normativ. Das autonome ästhetische Subjekt ist in ihm der Platzhalter des utopischen Projekts sozialen und individuellen Friedens, Platzhalter der Idee des absoluten Freiwerdens von Korrumpiertheit. Gesellschaft dagegen, gedacht als über Tauschwert und damit über ein Element des ökonomischen Systems organisiert, bedeutet Manipulation. Eine versöhnte Gesellschaft ist dann nur als negative Utopie vorstellbar. Nur innerhalb dieser Utopie ist jener Satz Adornos, nach Auschwitz sei Dichtung barbarisch, verstehbar. ${ }^{42}$ Er sagt, dass die Gesellschaft Dinge tut, die Kunst lächerlich erscheinen lassen, weil es durch diese Dinge vorstellbar wird, dass Gesellschaft irgendwann nicht mehr sei. Damit ergibt sich das Problem, die ungewisse Zukunft der Gesellschaft nicht näher bestimmen zu können und ihre Korrumpiertheit immer voraussetzen zu müssen. ${ }^{43}$

\footnotetext{
${ }^{41} \mathrm{Vgl}$. zum Konzept der Resonanz in der performativen Kunst und zu ihren ethischen Konsequenzen Mersch, Ereignis und Aura. S. $295 \mathrm{ff}$.

${ }^{42}$ Der Satz Adornos findet sich in Theodor W. Adorno, „Kulturkritik und Gesellschaft“, in: ders., Gesammelte Schriften in 20 Bd., hrsgg. v. R. Tiedemann, Bd. 10/1: Kulturkritik und Gesellschaft I, Frankfurt/Main 1977, S. 30

${ }^{43}$ Vgl. zum Vorwurf der Zukunftslosigkeit von Gesellschaft im dialektischen Denken Niklas Luhmann, Die Kunst der Gesellschaft, Frankfurt/Main 1995, S. 471
} 
Genau gegen diese Problemlage wendet sich die Systemtheorie. Sie hat die Selbstreferentialität der gesellschaftlichen Systeme ins Zentrum ihrer Überlegungen gestellt. ${ }^{44}$ Das gilt entsprechend natürlich auch für die Kunst, die, nachdem sie sich aus ihrer Abhängigkeit von Kirche, Mäzenen und anderen Repräsentanten politischer und ökonomischer Macht befreit hat, nur noch durch sich selbst beeinflusst wird. Autonomie kann dann nur noch heissen: Selbstlegitimation dessen, was in ihr produziert und kommuniziert wird, und Selbstbeobachtung dieser Produktion, denn nur durch die Referenz auf andere Kunstwerke kann man das beobachtete Artefakt als Kunstwerk erkennen. ${ }^{45}$ D.h. es gibt text- und bildimmanente Gesetzlichkeiten, also bindende Unterscheidungen, die ausschließlich für Literatur, bildende Kunst und Musik gelten und nur von ihnen weiterentwickelt werden können. Seit Mallarmé und dem Schritt zur Abstraktion in der Malerei bezieht sich die Kunstsprache nicht mehr auf etwas ausser ihr Liegendes, sie wird gegenstandsunabhängig. Lebensweltlich Deutbares wird so weit wie möglich eliminiert. ${ }^{46}$ Kunst wird non- oder selbstreferentiell.

Zugleich damit scheint Kommunikation verweigert zu werden. Die Werke werden dunkel und spröde, sie verschließen sich, sie wollen nicht, dass man einen identifizierbaren Sinn aus ihnen zieht. Sie kommunizieren nur noch sich selbst als Form und Ausdruck, nicht mehr als spezifischen Inhalt. Das ruft dann die sogenannten sekundären Diskurse auf den Plan, die Kommentatoren und Kunstkritiker, die die Aufgabe übernehmen, Anschlüsse für Sinnfortschreibungen herzustellen.

Wie wir bei Gerzens Sozialer Plastik gesehen haben, ist die heutige Kunst aber durchaus in der Lage, aussenstehende Beobachter ins Machen des Werks aktiv mit einzubeziehen. Kunst hat also einen derartigen Grad an Autonomie erreicht, dass sie produktive Operation, Selbstreflexion und Kommentar verknüpfen und in eine Aktion integrieren kann. Sie ist damit auch in der Lage, ihre Selbstnegation zu integrieren. Wenn die Avantgarden das Ende der Kunst inszenieren und diese Inszenierung wiederum als Kunst wahrgenommen wird, wenn in der Aktion von Gerz ein Teilnehmer sagt: „Ich will, dass die Kunst aufhört!“, und dies als Äußerung in die Aktion eingeht, dann ist eine neue Stufe der Selbstbeschreibung der Kunst erreicht.

Aus dieser Perspektive ist es völlig unmöglich, der Gesellschaft, wie bei Adorno, die Rolle des Manipulierenden zuzuschieben und dem künstlerischen Subjekt die Rolle des Platzhalters der Aufklärung, des Platzhalters für eine reine Selbstbestimmung. Aus dieser Perspektive ist

${ }^{44}$ Zur Selbstreferentialität als zentraler Kategorie in der Beschreibung des Kunstsystems bei Luhmann siehe Markus Koller, Die Grenzen der Kunst. Luhmanns gelehrte Poesie, Wiesbaden 2007, S. 20-97

45 „Die moderne Selbstbeschreibungsgeschichte des Kunstsystems von der Romantik über die Avantgarde bis zur Postmoderne läßt sich unter einem Gesichtspunkt zusammenfassen - als Variation zu einem Thema. Es geht in all diesen Fällen um die Behandlung der Vergangenheit in einem autonom gewordenen Kunstsystem und damit in allen Fällen um die Frage, wie Vergangenheit mit Zukunft, wie Gedächtnis mit Freiheit zum Seitenwechsel in allen Unterscheidungen vermittelt werden kann." (Niklas Luhmann, Die Kunst der Gesellschaft, Frankfurt/Main 1995, S. 489) Siehe weiter auch Niklas Luhmann, „Ausdifferenzierung der Kunst“, in: ders., Schriften zu Kunst und Literatur, hrsgg. v. N. Werber, Frankfurt/Main 2008, S. 410

${ }^{46} \mathrm{Zu}$ dieser Tendenz am Beispiel moderner Lyrik siehe Niklas Luhmann und Peter Fuchs, „Vom schweigenden Aufflug ins Abstrakte: Zur Ausdifferenzierung der modernen Lyrik“, in: dies., Reden und Schweigen, Frankfurt/Main 1989, S. $138-177$ 
es auch unmöglich, eine so rigide Trennung zwischen autonomer und korrumpierter Kunst zu setzen, wie Adorno es z.B. in der Philosophie der Neuen Musik tut, wo Schönbergs Musik Fortschritt bedeutet und die Schuld der Welt auf sich nimmt, Stravinsky dagegen reaktionär ist und seine Musik als pathologisch bezeichnet wird. ${ }^{47}$

Zugleich wird Kunst verstehbar als ein Übersetzungsnetzwerk aus spezifischen Kommunikationen. Über Produktion, Kritik, Zitat und Kommentar ergibt sich ein Beziehungsgeflecht unterschiedlicher Beobachtungen dessen, was dann als Kunst wahrgenommen und fortgeschrieben wird. Das macht das Aktuelle der oben erwähnten Gerzschen Aktion aus: Sie nimmt dieses Spiel der Kommunikationen in sich auf und macht dem Publikum klar, dass es sich nicht mehr auf die alte Rolle des neutralen oder unbeteiligten Betrachters zurückziehen kann. Niklas Luhmann hatte bereits sehr früh $(1974)^{48}$ darauf hingewiesen, dass Kunstkommunikation die Einheit von Produzent und Rezipient voraussetzen muss. In einer Zeit, in der selbst Kunsttheoretiker und Kunsthistoriker alles nur im Vorbehalt gegen die eigene Aussage legitimieren können, sind die Kommentatoren und die mehr oder wenig informierten Betrachter dazu aufgefordert, sich in das Spiel, d.h. in das Fortschreiben der Kunst, einzubringen.

Als kritische Frage bleibt: Was kann die Kunst der Gesellschaft sagen, wenn sie alles nur sich selbst sagt und in ihr keine Wertzurechnungen mehr möglich sind? Wie muss dann unsere Kommentarkultur aussehen, damit wir aus der Kunst Lehren ziehen können, die gesellschaftliche Dysfunktionalitäten wie soziale Spannungen und interkulturelle Diskrepanzen im Globalisierungsprozess vermeiden helfen? Aber es gibt auch noch eine weitere kritische Frage: Wenn die Systemtheorie Kunst als selbstreferentielles System beschreibt, das seine eigenen Disjunktionen und Kriterien festlegt, wie läßt sich dann überhaupt noch die Idee des gesellschaftlichen Progresses und der sozialen Gerechtigkeit fortschreiben? Die Systemtheorie hat durch ihr begriffliches Instrumentarium eine komplexere und zugleich doch einheitliche Beschreibung von Gesellschaft möglich gemacht. Andererseits ist in ihr das polemische Element, im positiven Sinne des Wortes, eliminiert worden. Vielleicht ist aber eben dieses Element von Adornos Ästhetik zu retten. Denn es fragt sich am Ende, ob wir ohne die Idee einer versöhnten Gesellschaft und ohne die Idee, dass die Kunst noch einen schwachen Widerhall des Versöhnten liefert, auskommen. Genauso fraglich ist allerdings auch, ob die Idee eines permanenten Übertragungsprozesses von Sinn- und Ordnungselementen ohne den Horizont einer universalen Verständigung auskommt, also ohne die Vorstellung, dass das babylonische Sprachengewirr irgendwann aufhört zu exisitieren.

\footnotetext{
${ }^{47}$ Siehe Theodor W. Adorno, Philosophie der neuen Musik, Gesammelte Schriften Bd. 12, Frankfurt/Main 2003

${ }^{48}$ Vgl. „Ist Kunst codierbar? - Ausschnitte aus der Diskussion“, in: Luhmann, Schriften zur Kunst und Literatur, S. 97
} 